\title{
Multiple myeloma and physical activity
}

\author{
Catherine S. Y. Lecat ${ }^{1 *}$, Orla McCourt ${ }^{1,2}$, Joanne Land ${ }^{3}$, Kwee Yong $^{1}$ and Abigail Fisher ${ }^{3}$
}

\begin{abstract}
Objective: Physical activity has been shown to improve quality of life in cancer patients with some evidence in multiple myeloma. This study aimed to determine myeloma patients' exercise levels, their perception of physical activity, and to explore correlations with quality of life. Myeloma outpatients were invited to complete a number of questionnaires, including the Godin leisure-time exercise questionnaire (GLTEQ) to determine their exercise levels, the Functional Assessment of Cancer Therapy-General (FACT-G) questionnaire to assess health related quality of life, and the Functional Assessment of Chronic Illness Therapy-Fatigue (FACIT-F) questionnaire to assess fatigue.

Results: Of the 65 respondents, $75 \%$ would like to increase their exercise level. Weakness, fatigue and pain were the most commonly perceived barriers to physical activity. 59\% would like to receive physical activity advice. Only 25\% were deemed active based on their GLTEQ scores. Finally, there was a significant positive correlation between the GLTEQ score and the FACT-G score $(p<0.001)$. Results highlight an unmet exercise need in myeloma patients. Current practice should be reviewed to develop a more holistic care model that incorporates tailored exercise advice or programme.
\end{abstract}

Keywords: Multiple myeloma, Physical activity, Exercise

\section{Introduction}

Multiple myeloma (MM) is an incurable bone marrow malignancy with multiple relapses and remission periods, followed by eventual treatment resistant disease. Recent novel therapies have dramatically improved patients' survival. MM survivors now face the challenge of regaining premorbid psychosocial and physical wellbeing, whilst coping with disease symptoms and treatment side effects. With osteolytic bone destruction occurring in nearly $90 \%$ of patients [1], physical activity (PA) is often perceived as increasing risk of injury and is not actively promoted. The lack of PA, along with long term toxicity from complex treatment regimens and autologous stem cell transplantation (ASCT), can lead to muscle atrophy, fatigue and de-functioning, thus negatively impact on quality of life (QoL) [2].

*Correspondence: Catherine.lecat@nhs.net

1 University College London Cancer Institute, 72 Huntley Street, London WC1E 6DD, UK

Full list of author information is available at the end of the article
PA has been shown to associate with less fatigue, improved QoL and physical functioning in cancer patients [3-5]. Previous studies confirmed that tailored exercise programme in MM patients was safe and feasible, with high adherence, and was associated with benefits in QoL, fatigue and muscle strength [6, 7]. Additional studies are required to further confirm these benefits. Moreover, a better understanding of the prevailing attitudes toward PA in patients and clinical teams is needed if such programmes are to become part of survivorship care, and to be supported by healthcare professionals (HCP).

PA is recognised as an important aspect to the quality of cancer patients' survival. The U.K. Independent Cancer Taskforce recommended that all cancer patients should receive tailored PA advice. In MM, such an initiative remains hampered by a lack of understanding and awareness of the benefits of PA, and how to promote safe exercise in the context of myeloma bone disease. We sought to find out MM patients' attitude towards PA and 
ascertain their levels of PA and QoL in order to better understand their survivorship needs.

\section{Main text \\ Methods}

This is a service evaluation study conducted in the University College London Hospital, a tertiary myeloma centre. Patients attending MM outpatient clinics were invited to complete a survey (see Additional file 1), collecting demographic data and information on their MM status and treatment. PA questions include whether they would like to increase their exercise level, their perceived barrier(s) to PA, whether they would like to receive PA advice and their preferred way(s) to receive it.

PA level was assessed using the Godin leisure-time exercise questionnaire (GLTEQ) $[8,9]$. A score of $>24$ represents physically active, 14-23 represents moderately active, and $<14$ represents insufficiently active. Functional Assessment of Cancer Therapy-General (FACT-G) and Functional Assessment of Chronic Illness TherapyFatigue (FACIT-F) questionnaires were used to assess QoL and fatigue respectively $[10,11]$.

Completion of this survey was optional and implied consent was obtained when patients returned the completed survey. All responses were anonymised. NHS Health Research Authority (HRA) ethics approval was not required based on the HRA decision tool [12] and the need for written consent was waived by HRA Research Ethics Committee (REC). Data were summarised descriptively and quantitative parameters were presented as percentages, medians, means and quartiles. Non-parametric Mann-Whitney U test and Kruskal-Wallis test were used to compare between groups. Associations between variables were analysed using the Spearman's correlation test. A p-value of $<0.05$ was deemed statistically significant. SPSS was used for statistical analyses and to generate figures.

\section{Results}

Between March and May 2019, 65 patients completed the survey. Patient characteristics are shown in Table 1. Two-third (66\%) were on myeloma treatment, with $26 \%$ on three or more lines. Median time from MM diagnosis was 3.8 years (range $0.1-15$ ). $12 \%$ had been diagnosed for over 10 years. $75 \%$ would like to increase their PA level and 9\% would not. The rest were either neutral or did not specify. The most commonly perceived barrier to improving PA was weakness (43\%), fatigue (40\%) and pain (26\%). Other barriers include neuropathy from myeloma treatment (20\%), myeloma bone disease (15\%), arthritis (9\%) and previous surgery limiting mobility (8\%). Parkinsonism and previous nerve injury were stated for two respondents. $71 \%$ reported that a combination of factors, rather than a single factor, prevented them from improving PA (Table 2).

Fifty-nine percent would like to receive PA advice from a HCP. The three most selected options to receive such advice were written leaflets (29\%), a face-to-face session with a $\mathrm{HCP}(28 \%)$ and verbal advice in clinic consultation (23\%). Other chosen methods include a telephone call from a HCP (14\%), mobile application (12\%) and internet website (11\%). None of them chose group seminar.

Our respondents had a median GLTEQ score of 15 (range 0-66). According to their scores, 25\% were deemed physically active, $17 \%$ moderately active and $39 \%$ inactive. Thirteen $(20 \%)$ did not specify their PA level. The median FACT-G score was 73.1 (range 31.2108 , mean $75.1 \pm 21.1$ ), and the median FACIT-F score of 32.5 (range $3-52$, mean $30.3 \pm 14.6$ ). GLTEQ, FACT$\mathrm{G}$ and FACIT-F scores were not significantly different between males and females, and between those on or off myeloma treatment. Nor did these scores correlate with years since myeloma diagnosis, or with numbers of prior treatment lines (Table 1). These scores did not differ significantly between age groups, apart from FACT$\mathrm{G}$, where the scores in 65-75 were higher than those in $45-54$ and in $>75 \mathrm{~s}(\mathrm{p}=0.049)$. Using a cut off FACITF score of 34[13], 49\% of the patients were classified as clinically fatigued. These clinically fatigued patients had a lower median GLTEQ score than those who were not $(\mathrm{p}<0.001)$.

Finally, there was a significant positive correlation between GLTEQ and FACT-G scores (Spearman's correlation coefficient $=0.62, \mathrm{n}=52, \mathrm{p}<0.001)$, and also between GLTEQ and FACIT-F scores (Spearman's correlation coefficient $=0.67, \mathrm{n}=52, \mathrm{p}<0.001)$ (Fig. 1).

\section{Discussion}

One key finding is the low PA level in our cohort of realworld MM patients. Although a different self-reported measure was used, our figure of $25 \%$ is much lower than the $67 \%$ of U.K. general population who were considered active (ages $75-84-53 \%$, age $85+-31 \%$ active) [14]. These low PA levels are hardly surprising; in addition to the fear of injury, related to their bone disease, MM patients suffer with a considerable symptom burden, related to their older age and co-morbidities, as well as treatment toxicities. Barriers to exercise are usually multifactorial, but neuropathy was featured in $20 \%$ of our participants, alongside more common cancerrelated symptoms such as fatigue and pain. Peripheral neuropathy is a common and often painful side effect of some of the commonest agents used to treat myeloma, including bortezomib and thalidomide. Similar to our findings, Craike et al. [15] reported that most barriers were related to MM symptoms and treatment toxicities. 
Table 1 Patient characteristics with the median, lower and upper quartiles of their GLTEQ, FACT-G and FACIT-F scores

\begin{tabular}{|c|c|c|c|c|c|c|c|}
\hline Patient characteristics $(n=65)$ & Frequency (\%) & GLTEQ score & p-value & FACT-G score & p-value & FACIT-F score & $p$-value \\
\hline \multicolumn{8}{|l|}{ Gender } \\
\hline Male & $38(58)$ & $15(3.8-31)$ & 0.92 & $70.7(58.1-94.1)$ & 0.56 & $31(16.8-40.3)$ & 0.99 \\
\hline Female & $26(40)$ & $12(0-36)$ & & $76.4(63.8-95.3)$ & & $34(17.5-44.7)$ & \\
\hline Not specified & $1(2)$ & & & & & & \\
\hline \multicolumn{8}{|l|}{ Age group } \\
\hline Less than 45 & $1(2)$ & Missing & 0.07 & 48 & 0.049 & 16 & 0.28 \\
\hline $45-54$ & $7(11)$ & $7.5(0-43.5)$ & & $63.3(37.4-86)$ & & $26(13-31)$ & \\
\hline $55-64$ & $18(28)$ & $12(7.5-23)$ & & $69.8(59.1-92.8)$ & & $35.5(20.5-43.8)$ & \\
\hline $65-75$ & $27(42)$ & $21(6-39)$ & & $84.4(69.5-100.7)$ & & $35(24-48)$ & \\
\hline Over 75 & $12(18)$ & $1.5(0-15.8)$ & & $65.4(46.8-90.9)$ & & $23(10-44.4)$ & \\
\hline \multicolumn{8}{|l|}{ Years since MM diagnosis } \\
\hline Less than 1 & $9(14)$ & $19.5(1.5-33.3)$ & 0.92 & $75(58.1-89.4)$ & 0.75 & $37(16-44.7)$ & 0.57 \\
\hline 1 to 5 & $28(43)$ & $15(6-34.5)$ & & $71.5(55.7-94)$ & & $31(14.25-39.8)$ & \\
\hline 6 to 10 & $14(22)$ & $15(3-36)$ & & $74.9(65.8-103.2)$ & & $35(24-49.3)$ & \\
\hline Over 10 & $8(12)$ & $12(0-58.6)$ & & $80.1(66.1-95)$ & & $34(23-43)$ & \\
\hline Not specified & $6(9)$ & & & & & & \\
\hline \multicolumn{8}{|l|}{ On myeloma treatment } \\
\hline Yes & $43(66)$ & $12(3-21)$ & 0.16 & $72(59.1-92.4)$ & 0.37 & $26.5(16.8-40.3)$ & 0.34 \\
\hline No & $21(32)$ & $26.5(6.8-51)$ & & 77.7 (63.5-98) & & $35(19.5-48.5)$ & \\
\hline Not specified & $1(2)$ & & & & & & \\
\hline \multicolumn{8}{|l|}{ Line of myeloma treatment } \\
\hline None & $7(11)$ & $25.5(0-42.3)$ & 0.43 & $78.4(65.3-104)$ & 0.72 & $39(23-50)$ & 0.30 \\
\hline Radiotherapy alone & $3(5)$ & 33 & & 66 & & 17 & \\
\hline $1 \mathrm{st}$ & $17(26)$ & $20(7.5-29)$ & & 68 (57.2-91.6) & & $34(24-39.5)$ & \\
\hline 2nd & $9(14)$ & $12(3-28)$ & & $76.5(46.6-97.8)$ & & $31(11.5-50)$ & \\
\hline 3rd or beyond & $17(26)$ & $9(0-15)$ & & $72.4(54.4-91.4)$ & & $23.5(14.5-37.8)$ & \\
\hline Not specified & $12(18)$ & & & & & & \\
\hline
\end{tabular}

p-values are displayed when comparing between independent groups

Table 2 Perceived barriers preventing patients from improving their level of physical activity

\begin{tabular}{ll}
\hline $\begin{array}{l}\text { Perceived barriers preventing patients from } \\
\text { improving their PA* }\end{array}$ & Frequency (\%) \\
\hline None & $16(25)$ \\
Weakness & $28(43)$ \\
Fatigue & $26(40)$ \\
Pain & $17(26)$ \\
Neuropathy from myeloma treatment & $13(20)$ \\
Known myeloma bone disease & $10(15)$ \\
Known arthritis & $6(9)$ \\
Previous surgery limiting mobility & $5(8)$ \\
Others & $2(3)$ \\
\hline
\end{tabular}

*Patients could choose more than one option. $71 \%$ of those who indicated barriers to PA reported that a combination of these factors, rather than a single factor, prevented them from improving their exercise levels
These factors inevitably have a negative impact on QoL, which is reflected by the lower mean FACT-G score in our cohort compared to that of the general population (75.1 versus 80.1) [16]. Interestingly, however, there was no meaningful difference in GLTEQ and FACT-G scores between those who were on and off myeloma treatment in this study. Furthermore, those who were 65-75 had higher FACT-G scores than two other age groups. These observations may be explained by the small sample size, and the multiple confounding physical and psychosocial factors that could influence these scores.

It is also noteworthy that while most patients had low exercise levels, the majority would like to improve this. This suggests an awareness of the possible benefits of exercise, and an unmet survivorship need. Over half of patients wished to receive PA advice from a HCP, suggesting that one of the barriers to exercising may be lack of confidence to do so safely. This echoes themes reported in existing literatures [6, 7], where patients were more confident to exercise under supervision by a 


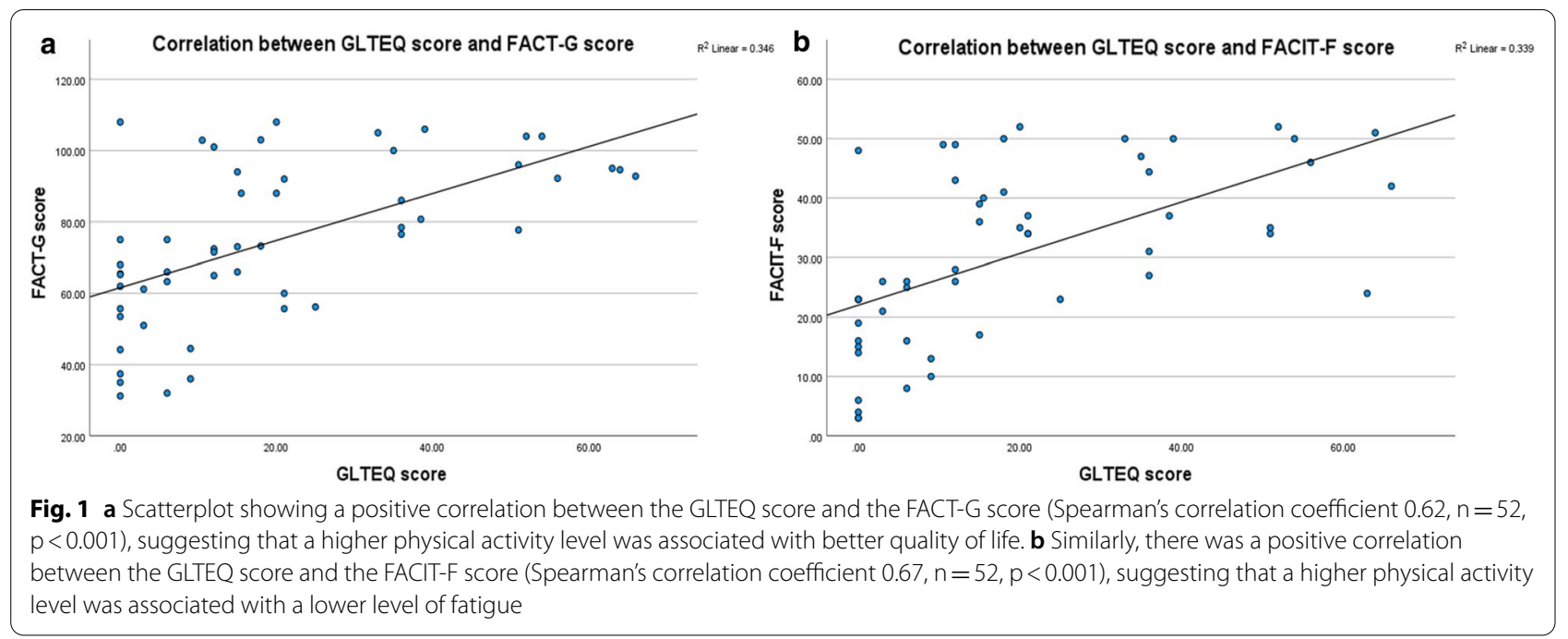

physiotherapist experienced in working in myeloma, and reflects the need for development of specialist physiotherapy workforce for this complex patient group. Oncology HCPs have also reported lack of clear guidelines and not being the right person to give PA and lifestyle advice [17].

Fatigue is a ubiquitous problem in cancer patients. In $M M$, it is compounded by the use of steroids in almost every treatment line, bone pain and fractures, and the autonomic neuropathy that accompanies many antimyeloma drugs. Our patients had a lower mean FACIT$F$ score than that of the general population (32.5 versus 43.6) [16]. Almost half (49\%) were classified as clinically fatigued (FACIT-F score <34) and unsurprisingly, they had significantly lower GLTEQ scores than those who were not fatigued $(\mathrm{p}<0.001)$. Patients with fatigue are often advised, or believe it is best, to rest to conserve energy. Excessive rest results in loss of functional capacity through deconditioning. Deconditioning and increasing effort to undertake daily activities, further exacerbates fatigue. Disrupting this cycle through exercise is key to its management [18]. Our study demonstrated an association between higher PA levels and lower fatigue. The well documented beneficial effects of exercise on cancerrelated fatigue, and on bone health, further highlights this unmet need in myeloma patients.

We observed that higher PA levels were associated with better QoL, in accordance with previous studies [3-5]. Although current study design does not allow a causal relationship to be concluded, existing evidence has shown that PA improves health related QoL [19]. Interestingly, for those who were physically active (GLTEQ score $>24, \mathrm{n}=16$ ), PA levels did not correlate with QoL (Spearman's correlation coefficient $0.21, \mathrm{p}=0.43$ ), nor did it with lower fatigue levels (Spearman's correlation coefficient $0.25, \mathrm{p}=0.35$ ). This could indicate a possible association threshold with PA and could potentially be used as a target activity level for patients to achieve.

MM patients have lifelong follow up in outpatient clinics, where emphasis is often placed on myeloma biochemical markers rather than patients' survivorship needs. Our study results highlight an unmet need to develop a more holistic care model, in which PA is assessed and promoted appropriately, with professional support from physiotherapists as appropriate. It is also important to identify and address barriers to PA proactively, such as through pain and neuropathy management. In line with MM supportive care guideline [20], regular PA should be encouraged from diagnosis to ensure that prehabilitation and rehabilitation are an integral part of every treatment line. To achieve this, it is key that both the patient and the clinical team are educated on the individual benefits and risks of exercise. To promote survivorship and selfmanagement, our team designed and runs a pilot multidisciplinary team (MDT) MM clinic involving a doctor, a nurse specialist and a physiotherapist, with wider MDT support if needed. Tailored exercise advice is given in each consultation and patients are encouraged to set achievable goals. They are signposted to various survivorship tools to help maintain physical and psychosocial wellbeing. Data on patient reported outcomes and patient experience are being collected to evaluate this alternative care model (REC Ref: 19/NS/0105).

A more formal way to promote PA is to deliver supervised exercise programmes, which, when administered by specialist personnel with appropriate screening procedures, have been shown to be safe and feasible [6]. Specialists should be involved so that MM related symptoms 
and treatment toxicities are taken into account in the prescription of individualised programmes. With limited literatures available, more high-quality, large randomised studies are needed to evaluate the impact of PA on different health parameters of MM patients. Research protocols such as PREeMPT [21] and PERCEPT studies [22], which look at prehabilitation before ASCT, will provide information to support for the use of exercise intervention in the future. Although such programmes may be a positive way to influence PA, only $28 \%$ of our patients preferred to receive PA advice in a face-to-face session. This suggests that formal exercise classes may not suit everyone, and clinicians need to devise flexible ways to promote PA depending on patients' preference, especially when digital healthcare technologies are becoming increasingly popular.

\section{Limitations}

- Results are limited by the cross-sectional nature of this single-site study, and by small sample size.

- Self-report data is subjected to bias and limitations such as introspective ability and interpretation of questions.

- Associations demonstrated in the study have no causal relationship due to its design.

\section{Supplementary Information}

The online version contains supplementary material available at https://doi. org/10.1186/s13104-021-05591-y.

Additional file 1. Physical activity survey

\section{Acknowledgements}

Not applicable.

\section{Authors' contributions}

$C L, O M, J L, K Y, A F$ designed the study. $C L$ performed the study and analysed the data. $C L, K Y$ wrote the paper. All authors read and approved the final manuscript.

\section{Funding}

This work is supported by RM Partners, who granted CL's clinical research fellowship (award code: 177569), and Takeda, who provided funding for the physiotherapy input in our pilot MDT myeloma clinic (award code: 19045). The funding bodies played no role in the design of the study and collection, analysis, and interpretation of data and in writing the manuscript.

\section{Availability of data and materials}

The datasets used and/or analysed during the current study are available from the corresponding author on reasonable request.

\section{Declarations}

Ethics approval and consent to participate In line with Health Research Authority (HRA) guidance, ethical approval was not required based on the HRA decision tool. Implied consent was obtained when patients returned the completed survey. The need for written consent was waived by the HRA Research Ethics Committee.

\section{Consent for publication}

Not applicable.

\section{Competing interests}

The authors declare no competing interests.

\section{Author details}

${ }^{1}$ University College London Cancer Institute, 72 Huntley Street, London WC1E 6DD, UK. ${ }^{2}$ University College London Hospitals NHS Foundation Trust, 235 Euston Road, London NW1 2BU, UK. ${ }^{3}$ University College London Behavioural Science and Health, Institute of Epidemiology \& Health, 1-19 Torrington Place, London WC1E 6BT, UK.

Received: 22 March 2021 Accepted: 28 April 2021

Published online: 07 May 2021

\section{References}

1. O'Donnell EK, Raje NS. Myeloma bone disease: pathogenesis and treatment. Clin Adv Hematol Oncol. 2017;15(4):285-95.

2. Jordan K, Proskorovsky I, Lewis P, et al. Effect of general symptom level, specific adverse events, treatment patterns, and patient characteristics on health-related quality of life in patients with multiple myeloma: results of a European, multicenter cohort study. Support Care Cancer. 2014;22(2):417-26.

3. Jones LW, Courneya KS, Vallance JK, et al. Association between exercise and quality of life in multiple myeloma cancer survivors. Support Care Cancer. 2004;12(11):780-8.

4. Shin WK, Song S, Jung SY, et al. The association between physical activity and health-related quality of life among breast cancer survivors. Health Qual Life Outcomes. 2017;15(1):132.

5. Servadio M, Cottone F, Sommer K, et al. Physical activity and healthrelated quality of life in multiple myeloma survivors: the PROFILES registry. BMJ Support Palliat Care. 2020;10(4):e35.

6. Groeneveldt L, Mein G, Garrod R, et al. A mixed exercise training programme is feasible and safe and may improve quality of life and muscle strength in multiple myeloma survivors. BMC Cancer. 2013;13:31.

7. Koutoukidis DA, Land J, Hackshaw A, et al. Fatigue, quality of life and physical fitness following an exercise intervention in multiple myeloma survivors (MASCOT): an exploratory randomised Phase 2 trial utilising a modified Zelen design. Br J Cancer. 2020;123(2):187-95.

8. Godin G, Shephard RJ. Godin Leisure-Time exercise questionnaire. Med Sci Sports Exerc. 1997;26(Suppl. 6):S36-8.

9. Godin G. The Godin-Shephard Leisure-time physical activity questionnaire. Health Fitness J Can. 2011;4(1):18-22.

10. Cella DF, Tulsky DS, Gray G, et al. The functional assessment of cancer therapy scale: development and validation of the general measure. J Clin Oncol. 1993;11(3):570-9.

11. Butt Z, Lai JS, Rao D, et al. Measurement of fatigue in cancer, stroke, and HIV using the functional assessment of chronic illness therapy-Fatigue (FACIT-F) scale. J Psychosom Res. 2013;74(1):64-8.

12. HHS Health Research Authority research decision tool. http://www.hradecisiontools.org.uk/research/ .2019. Accessed Mar 2019.

13. Van Belle S, Paridaens R, Evers G, et al. Comparison of proposed diagnostic criteria with FACT-F and VAS for cancer-related fatigue: proposal for use as a screening tool. Support Care Cancer. 2005;13(4):246-54.

14. NHS National Statistics on Obesity, Physical Activity and Diet 2020, https://digital.nhs.uk/data-and-information/publications/statistical/stati stics-on-obesity-physical-activity-and-diet/england-2020. Assessed July 2020.

15. Craike MJ, Hose K, Courneya KS, et al. Perceived benefits and barriers to exercise for recently treated patients with multiple myeloma: a qualitative study. BMC Cancer. 2013;13:319.

16. Cella D, Lai JS, Chang CH, et al. Fatigue in cancer patients compared with fatigue in the general United States population. Cancer. 2002;94(2):528-38. 
17. Williams K, Beeken RJ, Fisher A, Wardle J. Health professionals' provision of lifestyle advice in the oncology context in the United Kingdom. Eur J Cancer Care. 2015;24(4):522-30. https://doi.org/10.1111/ecc.12305.

18. Cramp F, Byron-Daniel J. Exercise for the management of cancer-related fatigue in adults. Cochrane Database Syst Rev. 2012;11:6145.

19. Marquez DX, Aguiñaga S, Vásquez PM, et al. A systematic review of physical activity and quality of life and well-being. Transl Behav Med. 2020;10(5):1098-109.

20. Snowden JA, Greenfield DM, Bird JM, et al. Guidelines for screening and management of late and long-term consequences of myeloma and its treatment. Br J Haematol. 2017;176(6):888-907.

21. Keen C, Skilbeck J, Ross H, et al. Is it feasible to conduct a randomised controlled trial of pretransplant exercise (prehabilitation) for patients with multiple myeloma awaiting autologous haematopoietic stem cell transplantation? Protocol for the PREeMPT study. BMJ Open. 2018;8(3):e021333.

22. McCourt O, Fisher A, Ramdharry G, et al. PERCEPT myeloma: a protocol for a pilot randomised controlled trial of exercise prehabilitation before and during autologous stem cell transplantation in patients with multiple myeloma. BMJ Open. 2020;10(1):e033176. https://doi.org/10.1136/bmjop en-2019-033176.

\section{Publisher's Note}

Springer Nature remains neutral with regard to jurisdictional claims in published maps and institutional affiliations.
Ready to submit your research? Choose BMC and benefit from:

- fast, convenient online submission

- thorough peer review by experienced researchers in your field

- rapid publication on acceptance

- support for research data, including large and complex data types

- gold Open Access which fosters wider collaboration and increased citations

- maximum visibility for your research: over 100M website views per year

At BMC, research is always in progress.

Learn more biomedcentral.com/submissions 